\title{
Neglected tropical diseases: exploring long term practical approaches to achieve sustainable disease elimination and beyond
}

\author{
Giuseppina Ortu* ${ }^{*}$ and Oliver Williams
}

\begin{abstract}
Background: Remarkable progress has been made in the fight against neglected tropical diseases, but new challenges have emerged. Innovative diagnostics, better drugs and new insecticides are often identified as the priority; however, access to these new tools may not be sufficient to achieve and sustain disease elimination, if certain challenges and priorities are not considered.

Main body: The authors summarise key operational challenges, and based on these, identify two major priorities: strengthening the capacity of the primary health care health system in correctly diagnosing and managing neglected tropical diseases; and establishing an effective disease surveillance process.

Five steps are proposed as concrete actions to build an effective primary health care service for neglected tropical diseases, and a health management information system capable of accurately reporting these diseases. Community engagement and formalization of community health workers role are proposed as essential components of these steps. Shift of financial support from disease oriented programmes to disease integrated interventions, improved access to international guidelines for primary health care staff, and availability of donated drugs in health care structures are also suggested as key elements of the proposed process.
\end{abstract}

Conclusion: The authors conclude that failure to address these priorities now may lead to further challenges on the long path towards neglected tropical disease elimination and beyond.

Keywords: NTDs, Primary health care, Integration, Surveillance, Community engagement

\section{Multilingual abstracts}

Please see Additional file 1 for translations of the abstract into the five official working languages of the United Nations.

\section{Background \\ NTD landscape}

Over the last few years there has been an increased interest in tackling neglected tropical diseases (NTDs), as their social, economic and health impact has become better known and more widely understood. As acknowledged diseases of poverty, NTDs have risen up the global public health agenda and eliminating NTDs by 2030 is now a target within the Sustainable Development Goals (SDGs) [1].

* Correspondence: g.ortu@malariaconsortium.org

Malaria Consortium, Development House, 56-54 Leonard street, London EC24 4LT, UK

(c) The Author(s). 2017 Open Access This article is distributed under the terms of the Creative Commons Attribution 4.0 International License (http://creativecommons.org/licenses/by/4.0/), which permits unrestricted use, distribution, and reproduction in any medium, provided you give appropriate credit to the original author(s) and the source, provide a link to the Creative Commons license, and indicate if changes were made. The Creative Commons Public Domain Dedication waiver (http://creativecommons.org/publicdomain/zero/1.0/) applies to the data made available in this article, unless otherwise stated.
Beyond the target itself, tackling NTDs is also critical to achieving Universal Health Coverage (UHC) and realising the Leave No-one Behind agenda, which have become two of the leading themes of the SDGs [2].

The World Health Organization (WHO) has developed clearly defined work plans for NTD elimination in Africa and Asia [3-5]. Through these, five intervention packages to prevent, control, treat and eliminate most NTDs have been identified:

- Preventive chemotherapy of population at risk/ infected

- Intensified case-finding and management

- Integrated vector control/management

- Provision of safe water, sanitation and hygiene

- Veterinary public health. 
Taking inspiration from the WHO NTD Roadmap [6], in 2012, donors, pharmaceutical companies, endemic countries and non-government organisations made the London Declaration commitment to end at least 10 NTDs by 2020 . Consequently, progress has been encouraging in several areas, such as the systematic mapping of preventive chemotherapy and transmission control NTDs (PCTNTDs) and the subsequent implementation of mass drug administration (MDAs).

However, as the NTD agenda has progressed, unsolved issues and new challenges have emerged, especially for PCT-NTDs, as have been highlighted by several NTD experts [7-10]. This year marks the fifth anniversary of the London Declaration, offering a fitting opportunity to examine the gaps and challenges facing NTD control and elimination efforts, to think about how to maintain what we have already achieved, and develop new and innovative approaches for managing all NTDs.

This paper summarises some of the practical unmet needs, challenges and assumptions in the current PCTNTD and (Innovative and) Intensified -Disease Management NTD (IDM-NTD) programmes. Concrete steps for moving existing interventions towards more sustainable and integrated NTD approaches are laid out, as well as suggestions on operational aspects that should be prioritized in future NTD interventions.

\section{(Main text)}

\section{Unmet needs, new challenges, and unspoken assumptions}

In the last few years, debates at international conferences [11-14] have been providing forums for a broad range of NTD stakeholders to discuss urgent unmet needs and explore new approaches to tackle emerging challenges related to the elimination and post-elimination phase. Debates have been focusing on MDA and post-MDA phase, mapping and monitoring disease transmission, community engagement, and disease morbidity management. Furthermore, whilst in the past the debate has focused mainly on PCT-NTDs, the new global drive to eliminate all NTDs requires a consideration of how best to integrate IDM-NTD within current NTD programmes and health structures, introducing new elements in the discussion such as how to roll out an integrated NTD surveillance work plan and integrated vector management. In Table 1, some of the most relevant unmet needs and new practical challenges are summarised, based upon these discussions, the literature and field experience. The table does not provide an exhaustive summary of the current practical challenges in the NTD elimination roadmap (already partially discussed by others $[7-10,15]$ ) but highlights some aspects of the key points in the debate.

Specifically, as previously highlighted by others $[9,10]$, and in Table 1, MDA presents several challenges for achieving NTD elimination. Whether countries decide to stop MDA because a disease burden has fallen significantly, or increase it to achieve elimination of a particular disease, the challenges would be very similar. This is because neither low burden nor eliminated diseases are likely to remain a public health priority, meaning that international financial support may stop and domestic financing may be redirected towards more urgent issues. In both cases, diseases could potentially bounce back to previous levels of prevalence.

Current debates have also highlighted the need to understand better how to engage the community in NTD interventions. Research has shown that community engagement, education and sensitisation are highly important for the effectiveness of public health interventions [16-18], as well the importance of initiating community based interventions, particularly for NTDs [19].

As an essential component of community engagement, community health workers (CHWs) play a key role in many NTD interventions [20]. However, as their workload has increased, the absence of corresponding and adequate technical and financial support has generated concerns about how to retain CHWs and sustain the quality of their services. The need for integrating these individuals into the primary health care (PHC) system has already been highlighted, specifically in onchocerciasis programmes [21]. Nevertheless, the role of CHWs in delivering health services has not been addressed adequately [22, 23], creating further obstacles to NTD elimination efforts.

Recent publications $[24,25]$ have also highlighted the urgent need to develop new rapid diagnostic tests (RDTs), drugs and insecticides, driven partly by the demand from NTD-endemic countries where these commodities would be deployed. However, for these commodities to be used routinely and effectively in a health care system, it would require:

- The presence of a health system with sufficient human resources, technical skills and adequate staff competencies to be able to engage immediately in the introduction of these commodities

- The acceptance of new approaches to diagnose and manage NTD patients

- The presence of a solid monitoring and evaluation framework to monitor the use of these new tools

- A functional disease surveillance process in place to provide routine data about disease burden and disease trends.

As Table 1 highlights, these requirements do not reflect the current situation in many low income countries, where there are often significant gaps in capacity 


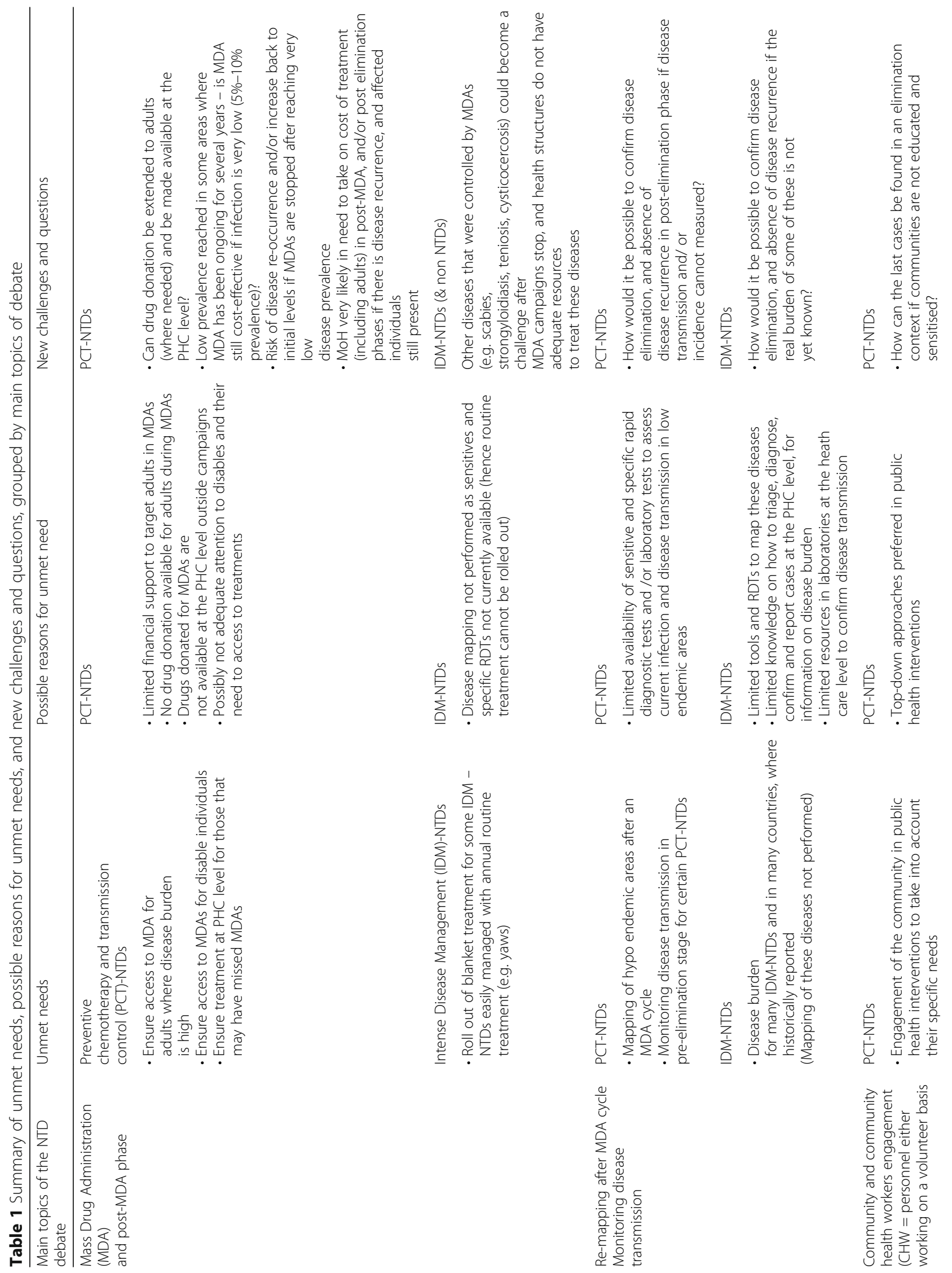




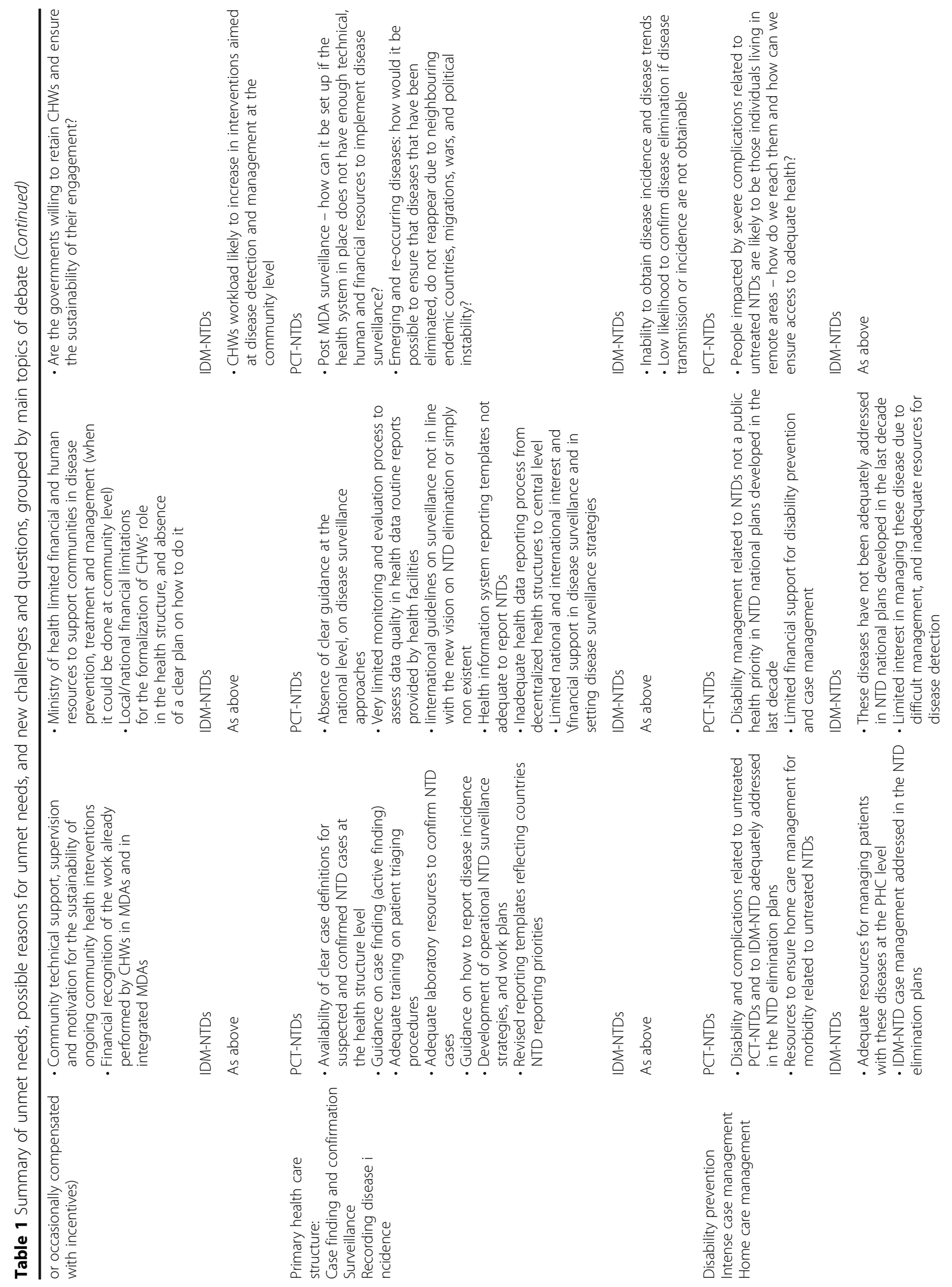




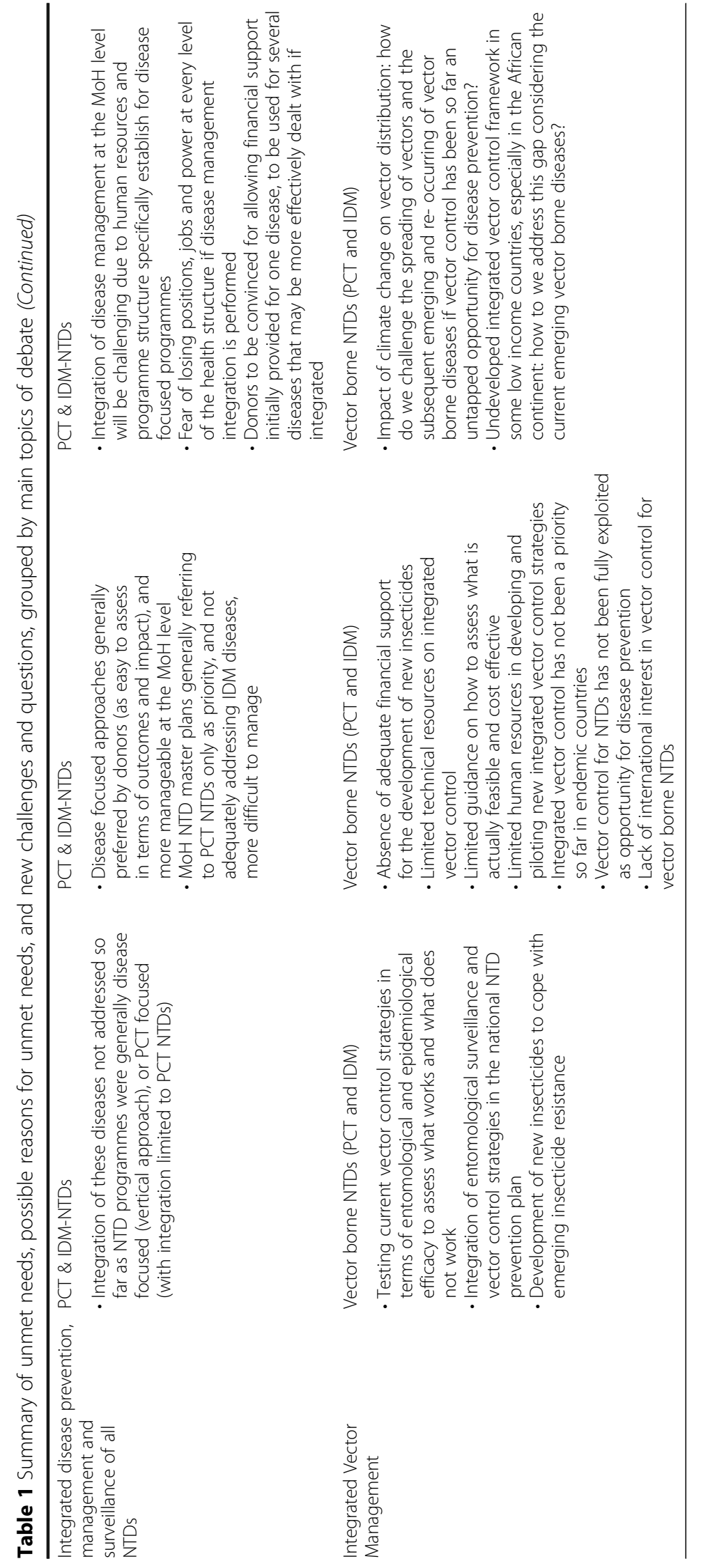


and technical knowledge at the health care delivery level [15]. The cost of using RDTs routinely in a primary health care setting may also be prohibitive, which could lead to governments using them solely as active surveillance tools to limit the overall cost. Such targeted use, however, does assume that there is some sort of surveillance strategy already in place for certain NTDs, which is very unlikely in many settings. Prioritising surveillance has been advocated by others in the sector [26-31], however very little has been done to ensure that this component of NTD management is included in NTD interventions.

Most of the current practical obstacles and unmet needs would not be completely resolved by the development and deployment of new commodities. An equally urgent priority is strengthening the capacity of the health care system and integrating NTD diagnosis, treatment and management so that communities can access health services via CHWs at the primary healthcare level. Routine disease surveillance should be established and integrated throughout the health system. Operational research is needed to understand how best to achieve this. Ideally it should be conducted before, or at least at the same time as, the introduction of new products.

Looking forward: operational steps and priority areas for NTD elimination and beyond

Figure 1 outlines five concrete steps to build sustainable NTD capacity in developing countries. Although these steps can overlap and be carried out concurrently, they have a logical flow and the risk of failing to follow them risks the mistakes of the past being repeated. If that happens, we are likely to find ourselves in the exact same situation in several years' time - with similar challenges and unmet needs, the re-emergence of NTDs that had been eliminated and a health system unable to cope. Participation of the community remains crucial to this process as explained below.

\section{The role of CHWs, community and education sector}

Community and CHW engagement is essential for the success of any PHC intervention. Chosen from within the community, CHWs are often the main point of contact with the formal health system for remote communities where health facilities are not easily accessible. They can play a fundamental role in disease prevention, control, management and surveillance.

To resolve the ongoing challenge of $\mathrm{CHW}$ retention, Ministries of Health could consider the option of offering fully-paid health professional roles to CHWs, or other paths to development. Such strategies have already been implemented by some African countries, through the introduction of monthly salaries or other performance based mechanisms [20,32-34]. The approach has also been suggested by WHO [22] for other health programmes that engage with CHWs. There is no single approach to formalising the role of CHWs, however it is important for them to receive routine training,

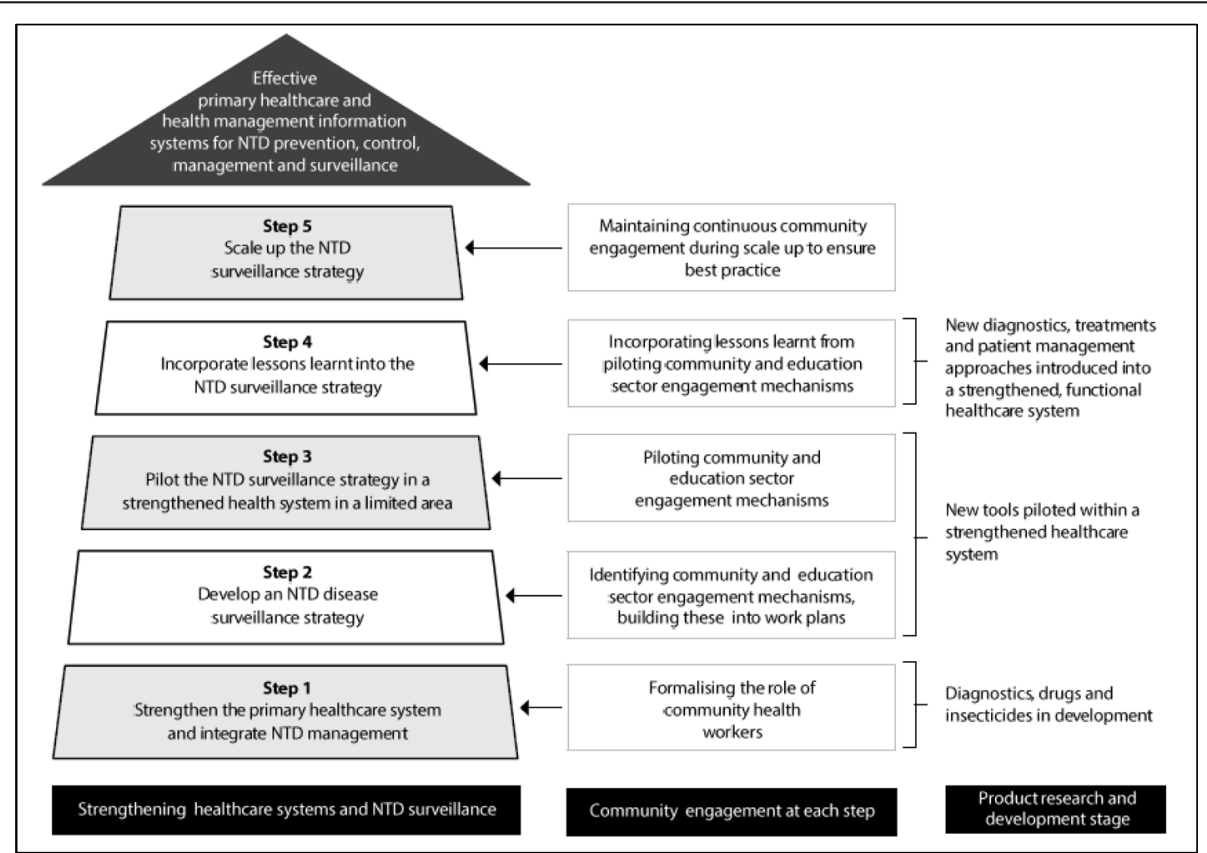

Fig. 1 Priority steps to be taken in the path towards NTD elimination and post-elimination. In this figure, three main areas are highlighted: health care system, community engagement and research and development, and how the community engagement and the research and development areas interact with the main priority (strengthening of the health care system) 
supportive supervision and constructive feedback. This could help ensure CHWs' satisfaction and retention in the long run, as well as build capacity in the health system. While the integration of disease management and strengthening of the health system is ongoing (Step 1), the formalisation of the role of CHWs should be a parallel activity.

Community and education sector engagement should begin during Step 2 (see Fig. 1) and continue throughout the process of building NTD capacity in the health system. The education sector, especially primary schools, play a central role in the life of rural communities and can therefore provide a useful connection to vulnerable groups, primarily children and mothers. Schools can provide a public space where community awareness about NTD prevention and control can be increased and disease-related disability and associated stigma discussed, understood and accepted. Engagement of this sector can reinforce community engagement by ensuring that messages delivered are consistent and reiterated in schools and other community contexts. Schools could be involved in many public health interventions targeting NTDs, such as routine MDAs, educational projects focusing on waste management and water storage for prevention of vector borne diseases, and school activities that teach hygiene and sanitation to help prevent worm infections and other diseases related to poor hygiene practices.

During Step 2, when the NTD surveillance strategy is being drafted, mechanisms should be built in to encourage meaningful and effective community engagement, making use of $\mathrm{CHW}$ networks. These mechanisms for engagement should then be tested and refined as part of Step 3.

\section{Step 1: Integrating NTD management and strengthening the primary healthcare system}

The first step is to shift from vertical approaches, in which one disease is tackled at a time, to a more horizontal system where disease management (including all aspects of management, e.g. prevention, diagnosis, treatment and surveillance) is integrated - not only for different NTDs but also with other communicable and noncommunicable diseases.

So far, integration has been applied for MDAs (e.g. when several drugs for treating different NTDs are delivered at once) and to some extent, in disease mapping (e.g. schistosomiasis and soil-transmitted helminth infections). The need now is to adopt an integrated approach at the PHC level. CHWs could be the connection between the community and the health care facility, and support the health staff in the routine implementation of these integrated preventive interventions. Health facilities could have a pivotal role in supporting the community in integrated vector surveillance and control [35] to prevent several NTDs that are carried by the same vector (e.g. Aedes aegypti control for dengue and chikungunya), or different vectors that have a similar behavioural pattern and can be controlled with a single strategy $[36,37]$.

The integration of disease control and management at the PHC level could be implemented for all diseases that have similar symptoms, such as NTDs that cause skin conditions. This approach would involve developing user-friendly triage tools that enable health staff at the PHC level to recognise diseases, and differentiate between them. Several publications have already highlighted examples of how the diagnosis and treatment of both NTDs and non-NTD skin-related diseases can be combined, and the benefits of doing so [38, 39]. It should be noted that grouping diseases that have very similar early signs, symptoms and morphology would not indicate a return to more vertically-oriented programmes. Rather, it would help to define more cost-effective approaches to managing NTDs aimed at supporting the PHC staff in their routine diagnosis of diseases that are hard to differentiate between. Taking into consideration existing levels of knowledge and limited resources, clinical (and pictographic) algorithms could be developed to start the process of addressing case detection, differential diagnosis and case referral for confirming suspected cases. Once these tools and processes are validated, training of health staff, especially at the PHC level, and the adequate provision of laboratory equipment, essential medicines and medical supplies for basic patient management should follow.

The benefits of strengthening the health system, especially at the first point of access, are for instance:

- Improved capacity for delivering routine disease prevention interventions at the community level (with the support of CHWs)

- Improved capacity for health facilities to take responsibility for routine NTD diagnosis and treatment through improved health worker knowledge, increased laboratory skills, and the provision of laboratory equipment and medicines

- An increased capacity for early NTD case detection, enabling health facilities to manage diseases more effectively, including cases that might recur even after elimination is achieved

- Improved reporting of NTD cases to the central level and quality of health data coming from decentralised health care facilities

During this step, it is important that the role of CHWs is formalised and their essential contribution to successful implementation is recognised, and as such, compensated. CHWs could have an important role in the following activities: 
- Encouraging community attendance during health campaigns (e.g. vaccinations and MDAs)

- Advocating for community-based morbidity management and acceptance of disabled individuals (e.g. those affected by elephantiasis and hydrocele)

- Supporting activities associated with disease prevention (e.g. integrated vector surveillance and control approaches, such as reducing vector breeding sites for dengue prevention or implementing specific hygiene practices to prevent worm infections)

As part of efforts to integrate NTDs with routine disease management activities at the health facility level, gaps in the health management information system (HMIS) reporting process would be highlighted during this first step. Challenges could include the absence of HMIS indicators for specific NTDs, or the use of indicators that are unable to distinguish between suspected and confirmed cases. These would be noticed at this step, and addressed to achieve a system in which a comprehensive set of reliable and relevant NTD indicators are included, and fully integrated into the HMIS reporting tools in use for other conditions and diseases. During this first step, NTD reliable data would be collected in order to provide the baseline NTD epidemiological information necessary to understand disease incidence. This would help to define epidemiological thresholds and identify areas where more resources should be invested in order to strengthen active disease monitoring and prevent outbreaks.

\section{Step 2: Developing an NTD surveillance strategy}

Once capacity has been built to correctly detect and confirm diseases, adequately manage and treat patients and routinely capture NTD incidence via HMIS reporting, the next step is to develop a disease surveillance strategy. Based on routinely collected data and country priorities in selecting which disease to report and notify, a surveillance strategy could be drafted. For instance, in areas bordering countries that are highly endemic for one specific NTD, sentinel sites to observe potential changes of disease trends could be set up. Likewise, in areas where a specific disease has not been reported, but the population is still exposed to environmental risks, sentinel sites may be needed as warning system for disease re-emergence.

The surveillance strategy should take into consideration available resources and national public health priorities. Eventually, the goal of this strategy would be to provide an efficient mechanism to monitor disease trends. The strategy should also contain practical approaches and processes to ensure that data collected at the community level is delivered to the central office for analysis in real time, providing governments with an accurate estimation of changing disease burdens with which to identify potential outbreaks and plan public health responses. This would also enable each subdistrict unit to define their own surveillance strategy for all relevant diseases.

NTD surveillance is crucial for disease control, elimination and post-elimination preparedness, and should therefore be the starting point for future NTD public health interventions. Starting with the already available diagnostic tools, drugs and expertise, it would be possible to improve the process of disease detection, confirmation, treatment and management.

During this step, CHWs also have an essential role to play, such as in supporting the mapping of diseases of interest (especially intense case management diseases such as Buruli ulcer) and establishing a communitybased surveillance system for early detection and reporting of suspected cases to primary health facilities (e.g. lymphatic filariasis detection [40]).

\section{Step 3: Piloting the NTD surveillance strategy}

Implementing an effective NTD surveillance system with concrete community engagement is not an easy task. There are various models and approaches, while local contexts require an additional layer of adaptation. Therefore it is important to pilot different models in defined areas of the country to assess their feasibility. In this phase, it is essential to outline a clear monitoring and evaluation (M\&E) framework to record input, process, output, outcome and impact indicators, and to ensure a quality assurance process throughout implementation. It is also highly advisable to establish an independent monitoring process to ensure data quality and reliability.

During this step, new drugs, RDTs and insecticides could be trialled and tested for effectiveness within a structure that has already been strengthened. Where the community is already engaged, this can also provide a clear indication of the viability of the new tools in the local context. The piloting process can take some time and the cost effectiveness of this step will have to be carefully assessed and incorporated into sustainability calculations with regards to the value for money NTD elimination provides.

CHWs would once again play an important role during step three, by supporting the piloting of proposed approaches, and, if needed, developing innovative, context-specific community-based solutions to combat NTD burdens.

\section{Step 4: Lessons learnt}

The lessons learnt from piloting each approach, including what worked, what did not and what could be 
improved upon, needs to be recorded and analysed. This analysis must include a cost evaluation for each model of surveillance. An important part of this process is sharing learning from localised pilot studies in different areas of a country so that approaches that fit a wide range of contexts can be developed, and a broad base of best practice evidence created.

Lessons learnt must be incorporated into the final NTD surveillance strategy prior to scale up, including any improvements that need to be made to the community and education sector engagement mechanisms. It is also paramount that the M\&E component, including routine supervision and performance assessment, as well as routine assessment of collected health data, is part of the final strategy.

\section{Step 5: Scale up}

The fifth and final step is to scale up the finalised NTD surveillance strategy to the national level. This will have financial, operational and political implications. By following the previous steps, there should be strong evidence demonstrating the effectiveness and value for money of the approaches, tools and processes included in the NTD surveillance strategy, which should provide the justification for the adoption of necessary national policies.

\section{Further considerations \\ International financial support}

The steps outlined above require some shift in terms of investment priorities. If financial investment has so far been dedicated to activities that are mostly single disease oriented, future investments should prioritise a set of interventions aimed at integrating NTD management within the PHC system, at least in those areas where the disease burden has already decreased and vertical programmes targeting specific diseases may no longer be cost effective. A shift towards an integrated approach, fully endorsed by donors, would also facilitate integration of diseases at the national level, especially where financially supported vertical health programmes may pose a challenge for Ministry of Health to operationalize this shift.

In the medium to long-term, funding from international donors will be necessary to build capacity and support the scaling up of effective strategies. There should then be a structured transition over time of financing responsibility from donors to national government, so that the services and capacity which have been painstakingly built are not lost due to budgetary constraints. This is particularly important when it comes to preserving mechanisms for community and education sector engagement, which are fundamental to the sustainability of any intervention.

\section{Monitoring and evaluation framework}

This shift towards integration requires a greater emphasis on M\&E which, despite being advocated for in the past [26], remains one of the main neglected aspects of the NTD agenda. Robust M\&E, with strong quality assurance mechanisms (including laboratory quality assessments) and the adequate supervision of processes to ensure collection of reliable health data, is a critical component throughout the five steps outlined above. If the impact of NTD interventions are to be measured and their value for money successfully assessed, then an effective M\&E framework is essential.

Roughly $5-10 \%$ of an intervention budget should be allocated to M\&E to measure the impact of that intervention [41] and inform the lessons learnt. An M\&E framework should include the routine recording of process, output, outcome and impact indicators, such as the number of detected and confirmed NTD cases, the number of health facilities where disease trends have triggered active epidemiological investigations, and the number of outbreaks detected early enough to trigger preparedness measures. It is important to note that a strengthened health system and an effective surveillance strategy may lead initially to an increase in the recorded disease incidence.

International stakeholders involved in supporting NTD activities in endemic countries, including NGOs and private sector contractors, should ensure routine assessment of the quality of the field work by adequately budgeting for data quality assessments and ensuring an independent monitoring of these activities. They should also support Ministries of Health to establish sustainable systems for routine PHC staff supervision and feedback in order to improve all aspects of patient management, and for health data collection for surveillance. Internal capacity in organisations supporting NTD activities should be adequate to provide effective technical and financial support for all M\&E activities.

\section{Drug donation}

An open discussion is needed about the possibility of introducing drug donations at the PHC level. Although medicines for individual treatment and care for IDM diseases have already been made available to health facilities, drugs for PCT- NTDs are currently donated for use in MDAs but not for routine PCT-NTD treatment. As part of current donation agreements, drugs left over from completed MDAs cannot be used at health facilities to treat patients, but must instead be kept for future campaigns. However, if financial support for subsequent MDAs is not available, treatments may expire and be wasted. This could be resolved with a routine drug distribution system to PHC centres based upon the number of cases reported. 


\section{Access to technical support}

There are many excellent international guidelines for NTDs that are publicly available, however some, such those on surveillance [42], need to be updated to include more recent NTD case definitions. Furthermore, the highly practical and user-friendly international guidelines on disease prevention, control and management [37, 43, 44] should be amended to include NTD reporting, if needed and as per country priorities, and made more accessible to PHC personnel, many of whom are in need of more technical support and guidance. Based on field observation, knowledge of the existence of these useful guidelines for health staff can be low, resulting in them being poorly adopted within health care structures especially in rural areas. More effective communication about the existence of these guidelines and facilitation in the access to these documents would help to ensure that these informative and useful guidelines reach the local level, where they are most needed.

\section{Aligning with the SDGs}

While above we have outlined the steps required to integrate NTDs into the health system and build a surveillance system, it is also important that the process is aligned with wider national and international efforts to strengthen health systems in pursuit of the SDGs [45]. Health system strengthening is receiving increased interest from donors as part of the growing momentum behind achieving UHC and the health SDG (SDG 3) - in which NTDs are explicitly mentioned (SDG3.3) [46]. The central pillars of UHC are extending universal access to highquality health services without causing financial hardship to users [47]. Clearly UHC cannot be achieved without a sustainable approach to eliminating NTDs that currently threaten 1.3 billion people and therefore they must be embedded within health system strengthening frameworks. Advocacy and engagement with donors and policy makers must focus on ensuring that the need to strengthen PHC for NTDs and NTD surveillance, and the supporting community engagement mechanisms, are included in this global agenda.

\section{Conclusion}

This paper highlights some of the operational challenges that global NTD elimination efforts face, and the practical steps that could be taken to move this agenda forward in a sustainable way. The five suggested steps, and the crucial community and education sector engagement needed throughout the process, will require a strong commitment from donors, national governments, NGOs and communities themselves. However, the long-term pay off will be strengthened NTD treatment and management services within the PHC system, hugely reduced NTD burdens and the capacity to respond to re-emerging and new threats alike. This is all possible with the technical knowledge, experience and tools that are already available, and can be achieved without creating parallel, unsustainable systems.

\section{Recommendations}

$>$ National governments could consider developing an integrated NTD prevention, control, management and surveillance strategy, using existing tools and knowledge. This could begin with strengthening the primary health care system, and initiating cross-talk and integration of vertically oriented programmes ongoing in the country. Disease-oriented donors could consider supporting this shift towards more integrated approaches for country benefit and programme sustainability.

$>$ National governments could strengthen community engagement through the formalisation of community health workers, ensuring that they are suitably supervised, trained, motivated and incentivised. The education sector could be engaged as a powerful link to the community.

$>5 \%-10 \%$ of the budget for NTD interventions could be allocated to setting up an M\&E framework in order to develop a better understanding of what works, what is more cost-effective and whether the intervention represents value for money. Within this framework, donors, NGOs and ministries of health would all have an essential role in ensuring provision of effective supervision, technical support, and quality assurance mechanisms to obtain reliable data at the primary health care level.

$>$ Pharmaceutical companies could consider donating drugs for routine NTD treatment and management at the primary health care level, and extending donation to treatment of adults, once capacity in confirming cases is built.

$>$ International health organizations and NTD experts could advice on how to improve the current health information management system reporting templates and international NTD surveillance guidelines in order to reflect current NTD reporting needs and elimination goals. Access and adherence of country health staff to already available and up-to-date guidelines for NTD prevention, control, management and surveillance could be improved.

$>$ Donors and national governments could agree a funding timetable, so that the responsibility for financing NTD prevention, treatment, management and surveillance is transitioned to national governments in a structured and sustainable manner. 


\section{Additional file}

Additional file 1: Multilingual abstracts in the five official working languages of the United Nations. (PDF 559 kb)

\section{Abbreviations}

CHW: community health workers; COR-NTD: Coalition for Operational Research on NTDs; HIMS: health information management system; IDM: intense disease management; M\&E: monitoring and evaluation; MDA: mass drug administration; NGDO: non-governmental development organizations; NTD: neglected tropical diseases: PCT: preventive chemotherapy and transmission control; PHC: primary health care; RDTs: rapid diagnostic tests; SDG: Sustainable Development Goals; UHC: Universal Health Coverage; WHO: World Health Organization

\section{Acknowledgements}

We thank Diana Thomas, Head of External Relations at Malaria Consortium, for her constructive and very valuable comments.

\section{Funding}

This work was supported by a UK Department for International Development Programme Partnership Arrangements grant.

\section{Availability of data and materials}

Not applicable for this manuscript.

\section{Authors' contributions}

GO drafted the manuscript and OW revised critically and finalized it. Both authors read and approved the final manuscript.

\section{Ethics approval and consent to participate}

There was no need of ethical approval and consent for the presented manuscript.

\section{Consent for publication}

The opinions as expressed belong to the authors do not necessarily reflect the views of the organization.

\section{Competing interests}

The authors have no competing interests in the manuscript.

Received: 14 June 2017 Accepted: 7 September 2017

Published online: 27 September 2017

\section{References}

1. United Nations. Sixty-ninth session of the United National General Assembly: Draft outcome document of the United Nations summit for the adoption of the post-2015 development agenda. New York: United Nations; 2013.

2. Fitzpatrick C, Engels D. Leaving no one behind: a neglected tropical disease indicator and tracers for the Sustainable Development Goals. Int Health. 2016;8(Suppl 1):i15-8. doi: 10.1093/inthealth/ihw002. PubMed PMID: 26940304; PubMed Central PMCID: PMCPMC4777229

3. World Health Organization. Regional strategic plan for neglected tropical diseases in the African Region 2014-2020. 2013.

4. World Health Organization. Regional strategic plan for integrated neglected tropical diseases control in South-East Asia region. 2011.

5. World Health Organization. Regional action plan for neglected tropical diseases in the Western Pacific (2012-2016). 2012.

6. World Health Organization - Accelerating work to overcome the global impact of neglected tropical diseases - a roadmap for implementation 2012.

7. Molyneux DH. The London Declaration on Neglected Tropical Diseases: 5 years on. Trans R Soc Trop Med Hyg. 2017; doi: 10.1093/trstmh/trw082. PubMed PMID: 28115685

8. Molyneux DH, Savioli L, Engels D. Neglected tropical diseases: progress towards addressing the chronic pandemic. Lancet. 2017;389(10066):312-25. doi: 10.1016/ S0140-6736(16)30171-4. PubMed PMID: 27639954

9. Bockarie MJ, Kelly-Hope LA, Rebollo M, Molyneux DH. Preventive chemotherapy as a strategy for elimination of neglected tropical parasitic diseases: endgame challenges. Philos Trans R Soc Lond Ser B Biol Sci. 2013;368(1623):20120144. doi: 10.1098/rstb.2012.0144. PubMed PMID: 23798692; PubMed Central PMCID: PMCPMC3720042

10. Webster JP, Molyneux DH, Hotez PJ, Fenwick A. The contribution of mass drug administration to global health: past, present and future. Philos Trans R Soc Lond Ser B Biol Sci. 2014;369(1645):20130434. doi: 10.1098/rstb.2013. 0434. PubMed PMID: 24821920; PubMed Central PMCID: PMCPMC4024227

11. Neglected Tropical Disease NGDO Network - A global forum for non governamental organizations working together on NTDs 2017. Available from: http://www.ntdngdonetwork.org/news-events/news/new-nnn-ntdsupplement-published-international-health. Accessed 22 Jan 2017.

12. Neglected Tropicall Diseases Support Centre - Breakout Summary Reports from COR-NTD 20152017 [22 Jan 2017]. Available from: http://www. ntdsupport.org/resources/breakout-summary-reports-cor-ntd-2015.

13. Neglected Tropical Diseases Support Centre 2014COR-NTD Meeting Presentations 2017 [22 Jan 2017]. Available from: http://www.ntdsupport.org/ resources/2014-cor-ntd-meeting-presentations

14. Neglected Tropical Diseaases Support Centre - Projects 2017. Available from: http://www.ntdsupport.org/projects. Accessed 22 Jan 2017.

15. Gyapong JO, Gyapong M, Yellu N, Anakwah K, Amofah G, Bockarie M, et al. Integration of control of neglected tropical diseases into health-care systems: challenges and opportunities. Lancet. 2010;375(9709):160-5. doi: 10.1016/S0140-6736(09)61249-6. PubMed PMID: 20109893

16. Bhutta ZA, Salam RA, Das JK, Lassi ZS. Tackling the existing burden of infectious diseases in the developing world: existing gaps and the way forward. Infect Dis Poverty. 2014;3:28. doi: 10.1186/2049-9957-3-28. PubMed PMID: 25105015; PubMed Central PMCID: PMCPMC4124963

17. Bhutta ZA, Sommerfeld J, Lassi ZS, Salam RA, Das JK. Global burden, distribution, and interventions for infectious diseases of poverty. Infect Dis Poverty. 2014;3:21. doi: 10.1186/2049-9957-3-21. PubMed PMID: 25110585; PubMed Central PMCID: PMCPMC4126350

18. Cyril S, Smith BJ, Possamai-Inesedy A, Renzaho AMN. Exploring the role of community engagement in improving the health of disadvantaged populations: a systematic review 2015. 2015;8. Epub 2015-01-05. doi: 10. 3402/gha.v8.29842.

19. Das JK, Salam RA, Arshad A, Maredia H, Bhutta ZA. Community based interventions for the prevention and control of Non-Helmintic NTD. Infectious Diseases of Poverty. 2014;3:24. doi: 10.1186/2049-9957-3-24. PubMed PMID: PMC4128610

20. Corley AG, Thornton CP, Glass NE. The Role of Nurses and Community Health Workers in Confronting Neglected Tropical Diseases in Sub-Saharan Africa: A Systematic Review. PLoS Negl Trop Dis. 2016;10(9):e0004914. doi: 10.1371/journal.pntd.0004914. PubMed PMID: 27631980; PubMed Central PMCID: PMCPMC5025105

21. Amazigo UV, Brieger WR, Katabarwa M, Akogun O, Ntep M, Boatin B, et al. The challenges of community-directed treatment with ivermectin (CDTI) within the African Programme for Onchocerciasis Control (APOC). Ann Trop Med Parasitol. 2002:96(Suppl 1):S41-58. PubMed PMID: 12081250

22. World Health Organization Regional Office for Africa Brazzaville Congo. Community Health Workers: Implications for large-scale CHW programs in the WHO African Region Health Systems and Services Cluster. Brazzaville Congo: World Health Organization; 2016.

23. Fleming FM, Matovu F, Hansen KS, Webster JP. A mixed methods approach to evaluating community drug distributor performance in the control of neglected tropical diseases. Parasit Vectors. 2016;9(1):345. doi: 10.1186/ s13071-016-1606-2. PubMed PMID: 27305942; PubMed Central PMCID: PMCPMC4910194

24. Barry MA, Murray KO, Hotez PJ, Jones KM. Impact of vectorborne parasitic neglected tropical diseases on child health. Arch Dis Child. 2016;101(7):640-7. doi: 10.1136/archdischild-2015-308266. PubMed PMID: 26921274

25. Hotez PJ, Pecoul B, Rijal S, Boehme C, Aksoy S, Malecela M, et al. Eliminating the Neglected Tropical Diseases: Translational Science and New Technologies. PLoS Negl Trop Dis. 2016;10(3):e0003895. doi: 10.1371/journal.pntd.0003895. PubMed PMID: 26934395; PubMed Central PMCID: PMCPMC4774924.

26. Baker MC, Mathieu E, Fleming FM, Deming M, King JD, Garba A, et al. Mapping, monitoring, and surveillance of neglected tropical diseases: towards a policy framework. Lancet. 2010;375(9710):231-8. doi: 10.1016/ S0140-6736(09)61458-6. PubMed PMID: 20109924

27. Bergquist R, Yang GJ, Knopp S, Utzinger J, Tanner M. Surveillance and response: Tools and approaches for the elimination stage of neglected tropical diseases. Acta Trop. 2015;141(Pt B):229-34. doi: 10.1016/j.actatropica. 2014.09.017. PubMed PMID: 25301340 
28. Liang S, Yang C, Zhong B, Guo J, Li H, Carlton EJ, et al. Surveillance systems for neglected tropical diseases: global lessons from China's evolving schistosomiasis reporting systems, 1949-2014. Emerg Themes Epidemiol. 2014;11:19. doi: 10.1186/1742-7622-11-19. PubMed PMID: 26265928; PubMed Central PMCID: PMCPMC4531518

29. Tambo E, Ai L, Zhou X, Chen JH, Hu W, Bergquist R, et al. Surveillance-response systems: the key to elimination of tropical diseases. Infect Dis Poverty. 2014;3:17. doi: 10.1186/2049-9957-3-17. PubMed PMID: 24971165; PubMed Central PMCID: PMCPMC4071800

30. Zhang Y, MacArthur C, Mubila L, Baker S. Control of neglected tropical diseases needs a long-term commitment. BMC Med. 2010;8:67. doi: 10.1186/ 1741-7015-8-67. PubMed PMID: 21034473; PubMed Central PMCID: PMCPMC2987894

31. Zhou XN, Bergquist R, Tanner M. Elimination of tropical disease through surveillance and response. Infect Dis Poverty. 2013;2(1):1. doi: 10.1186/20499957-2-1. PubMed PMID: 23849433; PubMed Central PMCID: PMCPMC3707090

32. Chilundo BG, Cliff JL, Mariano AR, Rodriguez DC, George A. Relaunch of the official community health worker programme in Mozambique: is there a sustainable basis for iCCM policy? Health Policy Plan. 2015;30(Suppl 2):ii5464. doi: 10.1093/heapol/czv036. PubMed PMID: 26516151; PubMed Central PMCID: PMCPMC4625760.

33. Condo J, Mugeni C, Naughton B, Hall K, Tuazon MA, Omwega A, et al. Rwanda's evolving community health worker system: a qualitative assessment of client and provider perspectives. Hum Resour Health. 2014; 12:71. doi: 10.1186/1478-4491-12-71. PubMed PMID: 25495237; PubMed Central PMCID: PMCPMC4320528

34. Retaining Community Health Workers in Ethiopia 2014. Available from: http// www.capacityplus.org/community-health-workers-ethiopia. Accessed 4 Sept 2015.

35. Bowman LR, Donegan S, McCall PJ. Is Denque Vector Control Deficient in Effectiveness or Evidence?: Systematic Review and Meta-analysis. PLoS Negl Trop Dis. 2016;10(3):e0004551. doi: 10.1371/journal.pntd.0004551.

36. Golding N, Wilson AL, Moyes CL, Cano J, Pigott DM, Velayudhan R, et al. Integrating vector control across diseases. BMC Med. 2015;13(1):249. doi: 10. 1186/s12916-015-0491-4.

37. World Health Organization. A toolkit for integrated vector mangement in sub-Saharan Africa. Geneva, Switzerland; 2016.

38. Engelman D, Fuller LC, Solomon AW, McCarthy JS, Hay RJ, Lammie PJ, et al. Opportunities for Integrated Control of Neglected Tropical Diseases That Affect the Skin. Trends Parasitol. 2016;32(11):843-54. doi: 10.1016/j.pt.2016. 08.005. PubMed PMID: 27638231

39. Mitja O, Marks M, Bertran L, Kollie K, Argaw D, Fahal AH, et al. Integrated Control and Management of Neglected Tropical Skin Diseases. PLoS Negl Trop Dis. 2017;11 (1):e0005136. doi: 10.1371/journal.pntd.0005136. PubMed PMID: 28103250

40. Stanton M, Molineux A, Mackenzie C, Kelly-Hope L. Mobile Technology for Empowering Health Workers in Underserved Communities: New Approaches to Facilitate the Elimination of Neglected Tropical Diseases. JMIR Public Health Surveill. 2016;2(1):e2. doi: 10.2196/publichealth.5064. PubMed PMID: 27227155; PubMed Central PMCID: PMCPMC4869228

41. Frankel N, Gage A. M\&E Fundamentals. A Self-Guided Minicourse - MEASURE Evaluation 2007.

42. World Health Organization and Centers for Disease Control and Prevention - Technical Guidelines for Integrated Disease Surveillance and Response in the African Region 2010.

43. World Health Organization, Regional Office for South-East Asia - Comprehensive guidelines for prevention and control of dengue and dengue haemorrhagic fever - Revised and expanded edition. 2011.

44. World Health Organization - IMAI District Clinician Manual: Guidelines for the management of common illnesses with limited resources - Hospital Care for Adolescents and Adults. 2011.

45. Bangert M, Molyneux DH, Lindsay SW, Fitzpatrick C, Engels D. The crosscutting contribution of the end of neglected tropical diseases to the sustainable development goals. Infect Dis Poverty. 2017;6(1):73. doi: 10.1186/ s40249-017-0288-0. PubMed PMID: 28372566; PubMed Central PMCID: PMCPMC5379574

46. SDG 3: Ensure healthy lives and promote wellbeing for all at all ages 2017. Available from: http://www.who.int/sdg/targets/en/. Accessed 9 Mar 2017.

47. Organization WH. Universal Health Coverage - what does it mean? (video) 2017. Available from: http://www.who.int/universal_health_coverage/en/. Accessed 9 Mar 2017.

\section{Submit your next manuscript to BioMed Central and we will help you at every step:}

- We accept pre-submission inquiries

- Our selector tool helps you to find the most relevant journal

- We provide round the clock customer support

- Convenient online submission

- Thorough peer review

- Inclusion in PubMed and all major indexing services

- Maximum visibility for your research

Submit your manuscript at www.biomedcentral.com/submit 